\title{
A universal deep learning-based framework towards fully ab initio simulation of atmospheric aerosol nucleation
}

Shuai Jiang ( $\square$ shuaijiang@ustc.edu.cn )

University of Science and Technology of China

Yi-Rong Liu

University of Science and Technology of China

Teng Huang

Chinese Academy of Sciences

Ya-Juan Feng

University of Science and Technology of China

Chun-Yu Wang

University of Science and Technology of China

\section{Zhong-Quan Wang}

University of Science and Technology of China

\section{Bin-Jing Ge}

University of Science and Technology of China

Quan-Sheng Liu

University of Science and Technology of China

Wei-Ran Guang

University of Science and Technology of China

Wei Huang

University of Science and Technology of China

Article

Keywords:

Posted Date: January 6th, 2022

DOI: https://doi.org/10.21203/rs.3.rs-1191188/v1

License: (c) (1) This work is licensed under a Creative Commons Attribution 4.0 International License.

Read Full License 
Version of Record: A version of this preprint was published at Nature Communications on October 14th, 2022. See the published version at https://doi.org/10.1038/s41467-022-33783-y. 
A universal deep learning-based framework towards fully ab initio simulation of atmospheric aerosol nucleation

Atmospheric aerosol nucleation contributes to around half of cloud condensation nuclei globally. Despite the importance for climate, detailed nucleation mechanisms are still poorly understood. Understanding aerosol nucleation dynamics is hindered by non-reactivity of force fields and high computational costs due to rare event nature of aerosol nucleation. Developing reactive force fields for nucleation systems are even more challenging than covalently bonded materials because of wide size range and high dimensional characteristics of non-covalent hydrogen bonding bridging clusters. Here we proposes a system transferable framework to train an accurate reactive force field (FF) based on deep neural network (DNN) and further bridges the DNN-FF based molecular dynamics (MD) with cluster kinetics model based on Poisson distributions of reactive events to overcome high computational costs from direct MD. We found that previously reported acid-base formation rates tend to be underestimated several times, emphasizing acid-base nucleation observed in multiple environments should be revisited.

Theoretical understanding of nucleation mechanism largely relies on classical nucleation theory $(\mathrm{CNT})^{1}$ originally proposed in 1935, which gives a general mind map for nucleation thermodynamics and kinetics ${ }^{2}$ even though the capillary assumption is extensively criticized ${ }^{3}$. Emergence $^{4}$ in 2011 and then broadly employed ${ }^{5-10}$ theoretical model, Atmospheric Cluster Dynamics Code (ACDC), surmounts the drawbacks of CNT through coupled quantum chemical thermodynamics ${ }^{11}$ with the birth-death equations ${ }^{2}$. Within ACDC, collision rate constants and evaporation rates are the two most critical parameters, determining the accuracy to predict macro parameters like cluster concentrations and formation rates which can be directly compared with experiments ${ }^{5}$. Evaporation rates, derived from detailed balance and ab initio thermodynamics, can be very accurate with sophisticated quantum chemical calculations ${ }^{12}$. However, collision rate constants, derived from simple hard sphere model, are still very rough, which accuracy is far from the $a b$ initio based evaporation rates'. Moreover, the accuracy of collision rate constants is extremely important especially for collision-controlled systems like sulfuric acid-dimethylamine system as evaporation rates are close to zero ${ }^{13}$. Pioneering work ${ }^{14}$ investigated the collisions between sulfuric acid monomers, however, the force field utilized there lacks reactivity and the computational costs of extending the method on more collisions among molecules and/or clusters are enormous. So highly accurate and computationally cheap reactive force field for flexible nucleation clusters is urgently needed to simulate nucleation processes with fully ab initio.

Here we propose a general framework to potentially boost the aerosol nucleation simulation toward fully ab initio. In the framework, comprehensive data sets are firstly prepared though passive learning coupled with active learning techniques. Then deep neural network based force field (DNNFF) is trained so that robust nucleation molecular dynamics (MD) simulations can be performed to derive the collision rate constants based on Poisson distribution. Then static quantum chemical thermodynamics based evaporation rates are coupled with DNN-FF based MD derived collision rate constants into cluster dynamics model to provide ab initio kinetics for simulating atmospheric aerosol nucleation. 


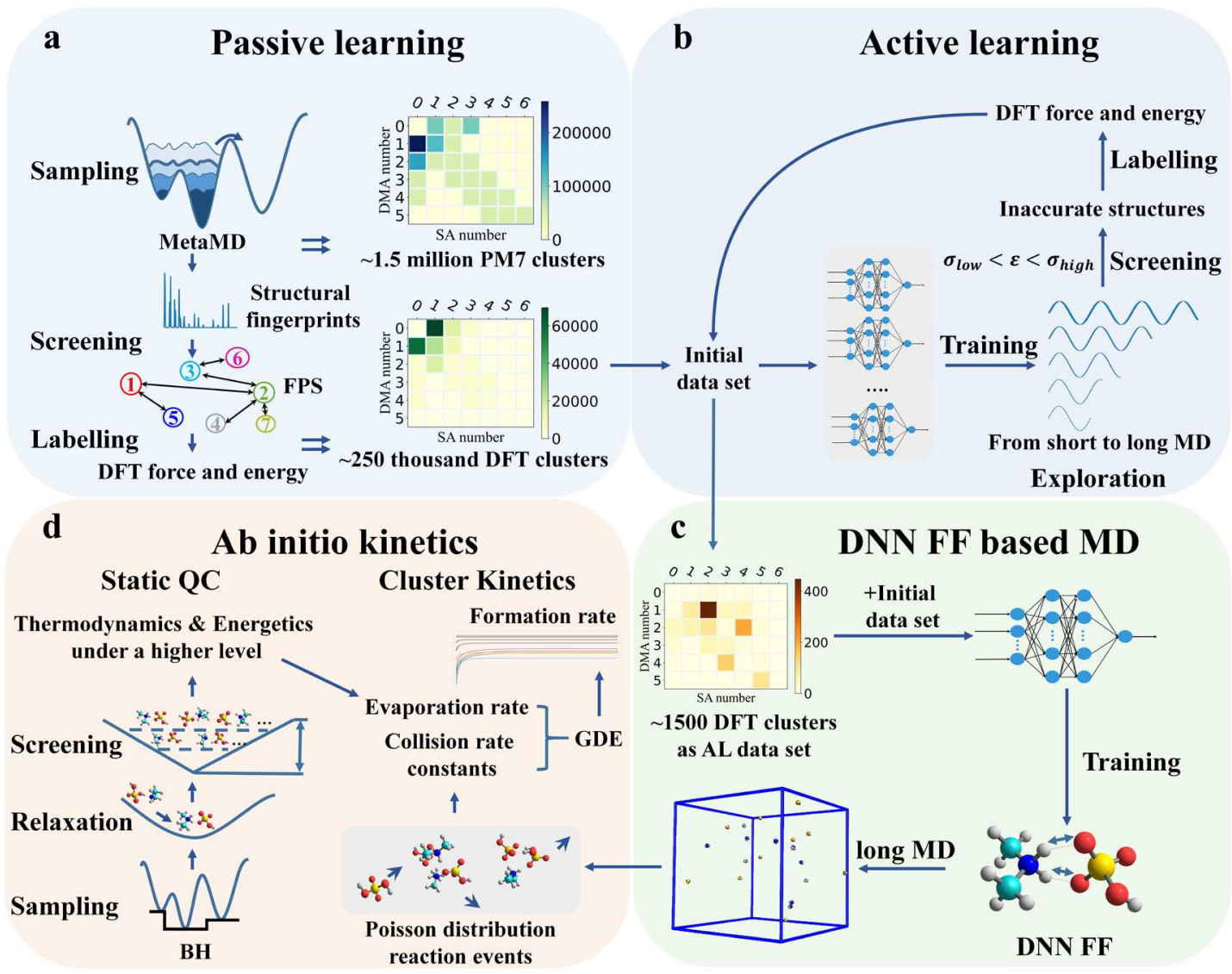

Figure 1 | A general framework towards fully ab initio simulation of atmospheric aerosol nucleation. $\mathbf{a}$ and $\mathbf{b}$ shows passive learning and active learning techniques to prepare data set for DNN respectively. c, DNN-FF driven MD. d, Cluster kinetics simulation based on MD derived collision rate constants and static quantum chemistry $(\mathrm{QC})$ calculation derived evaporation rates.

\section{Results}

A universal framework for fully ab initio simulation of aerosol nucleation. The key modules in the framework is shown in Fig. 1. The details in each module can be found in method section, so here the major points about significance and correlation for each module are given. Here passive learning is no doubt very important as the provided data set occupies most of the final data set (99.37 \% in Extended Data Table 3). However, there are two main shortcomings for passive learning. Firstly, cluster composition in each passive learning is set beforehand, so traversing all compositions for limited cluster size would undoubtedly produce redundant data set. Secondly, the sampling and subsequent screening structure number is empirical. Comparatively, active learning is very critical despite of the very small proportion of final data set $(0.63 \%$ in Extended Data Table 3$)$. To be noted, tests show purely based on passive learning provided data set cannot provide a robust DNN model as unphysical behaviors are observed, that's why active learning is introduced afterwards. The cluster size sampled by passive learning is based on the clusters stability characteristics that acidbase clusters are mostly stable when the difference between acid number and base number is less than or equal to one ${ }^{5}$. Active learning not only supplements the structures for passive learning sampled size but also points to the cluster compositions with high evaporation rates, e.g. (DMA) 4 as we can see from active learning data set in Fig. 1c, which can normally be ignored through passive learning. Collision rate constants, derived from MD simulations based on Poisson distribution 
reaction events (Fig. 1d), are essentially independent of cluster concentrations, making high concentration MD simulations valuable for further cluster kinetics.

Due to the interpolate nature of DNN, size scalability is still limited for extending only several molecules in this case. However, considering the rare event nature ${ }^{15}$ of aerosol nucleation, direct MD for ambient or laboratory aerosol nucleation with high concentration is very expensive ${ }^{16}$, challenging the application value of DNN trained model. We bridge the gap between micro parameters and macro ones through embedding MD derived rate constants based on Poisson distribution into cluster kinetics model. Those DNN-FF based MD derived constants coupling with static QC derived evaporation rates effectively boost the aerosol simulation towards fully ab initio.
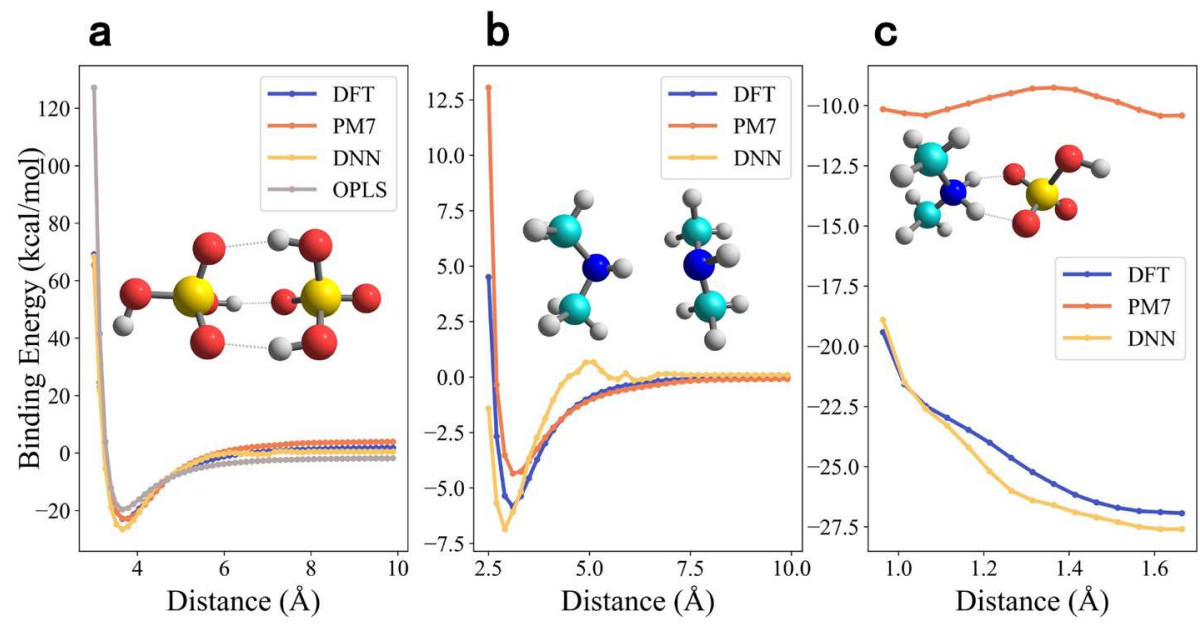

\section{d}
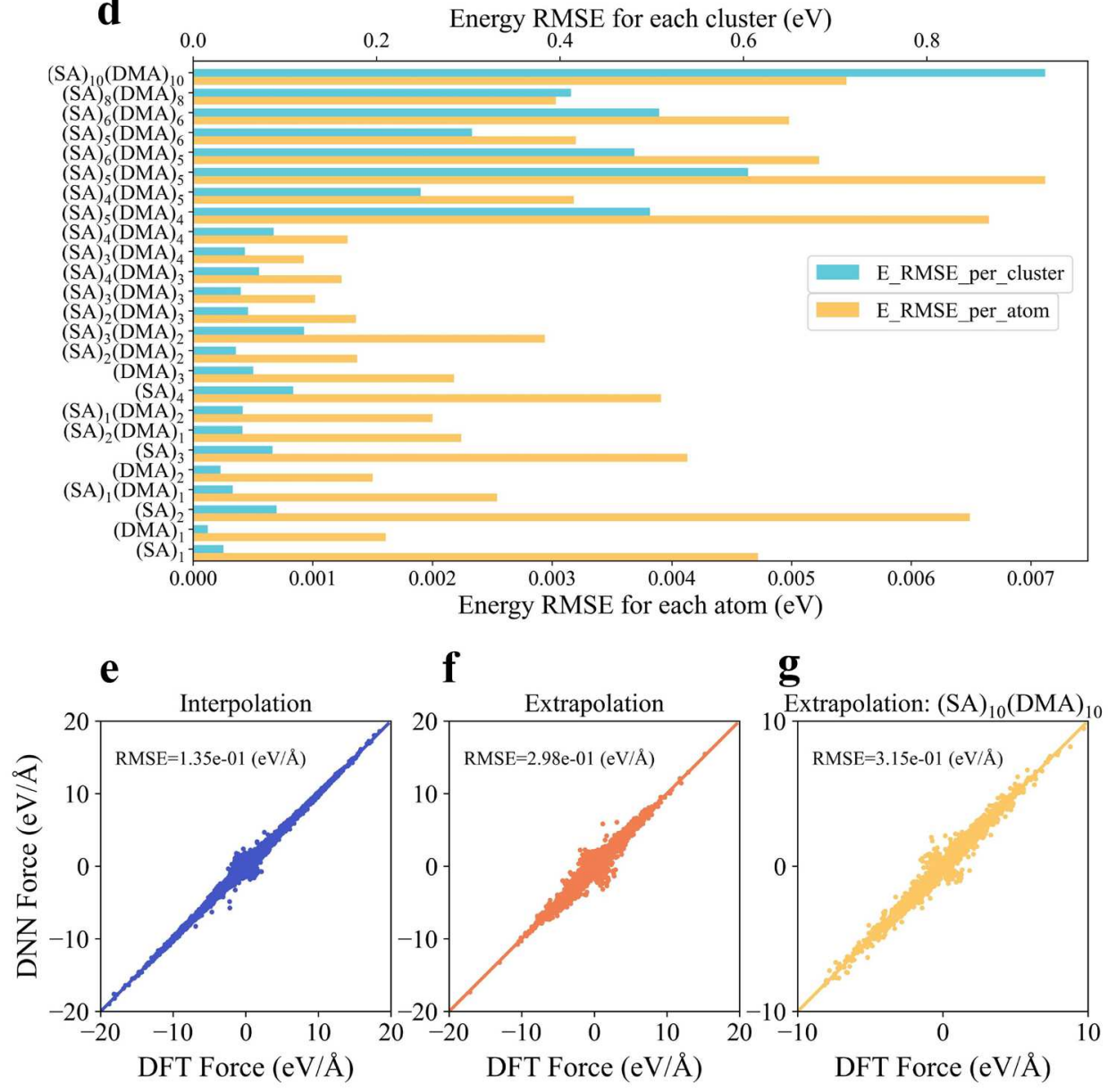
Figure 2 | DNN force field benchmark. $\mathbf{a}, \mathbf{b}$ and $\mathbf{c}$ give the dimer detachment curves for sulfuric acid-sulfuric acid (SA-SA), dimethylamine-dimethylamine (DMA-DMA) and sulfuric aciddimethylamine (SA-DMA), respectively. d, Energy root mean squared error (RMSE, in eV) for each cluster (in blue) or each atom within cluster (in yellow). e, Force interpolation $(\mathrm{eV} / \AA)$ ). f, Force extrapolation $(\mathrm{eV} / \AA)$. g. Force extrapolation $(\mathrm{eV} / \AA)$ for $(\mathrm{SA})_{10}(\mathrm{DMA})_{10}$.

The benchmark of DNN force field. The dimer detachment curves in Fig. 2a-c provide the basic picture about the performance of various force fields. For root mean squared error (RMSE) of binding energy (Extended Data Table 1), DNN is clearly superior than PM7 semi-empirical method $^{17}$ and optimized potentials for liquid simulation (OPLS) $)^{18}$. Notably, DNN performs largely better than OPLS for sulfuric acid (SA) dimer and PM7 for sulfuric acid-dimethylamine (SA-DMA) dimer. For smoothness, PM7 and OPLS is smoother than DNN generally, it is probably due to the absence of electrostatic interactions beyond the cutoff ${ }^{19}$, however, the novelty and validity of the proposed framework is not affected. Despite of the shortcoming, nanosecond MD simulation based on the DNN-FF is still working without observing unphysical behaviors like molecules dissociating into atoms. The cluster size resolved energy RMSE for each atom (Fig. 2d) shows high accuracy with the largest value lowing than $7 \times 10^{-2} \mathrm{eV}$. The maximum cluster size within the training set is $(\mathrm{SA})_{5}(\mathrm{DMA})_{6}$. To be noted, $(\mathrm{SA})_{\mathrm{m}}(\mathrm{DMA})_{\mathrm{n}}$ represents a cluster with $\mathrm{m}$ number of sulfuric acid molecules and $n$ number of dimethylamine molecules. The size extension to (SA) ${ }_{10}(\mathrm{DMA})_{10}$ gives quite good accuracy with $2.98 \times 10^{-1} \mathrm{eV} / \AA$ for all cluster sizes considered here (Fig. 2f) and $3.15 \times$ $10^{-1} \mathrm{eV} / \AA$ for $(\mathrm{SA})_{10}(\mathrm{DMA})_{10}$ alone (Fig. $2 \mathrm{~g}$ ). In a word, DNN model's superior performance in energy and force descriptions in addition to its distinguished size scalability lay a solid foundation for robust nucleation MD simulations. 
a
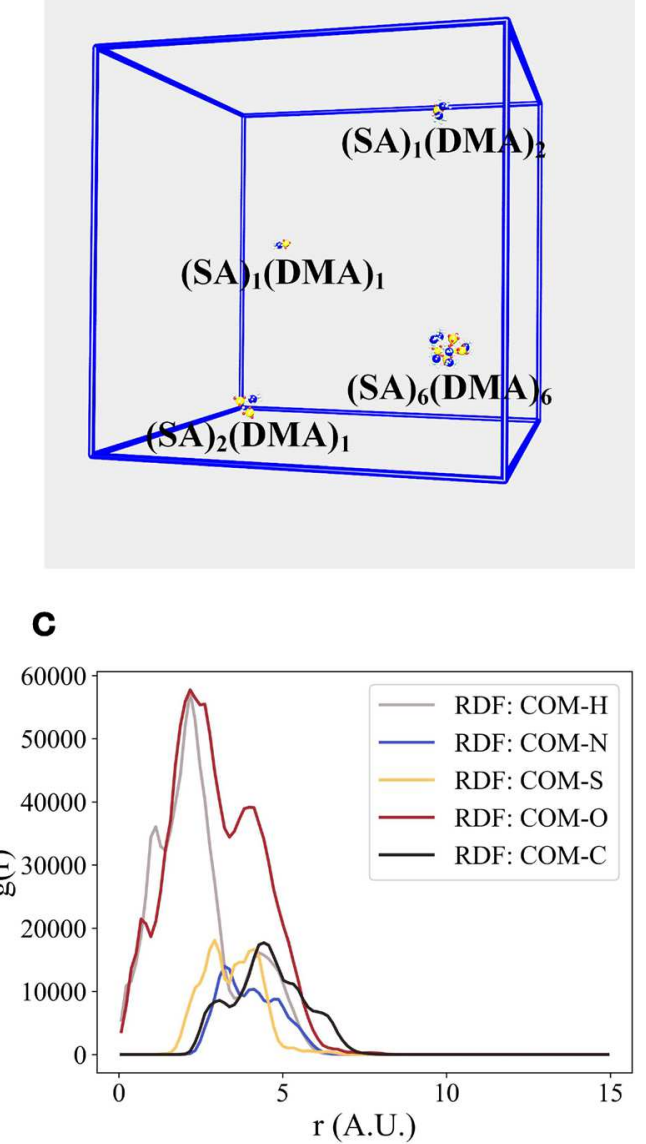

b

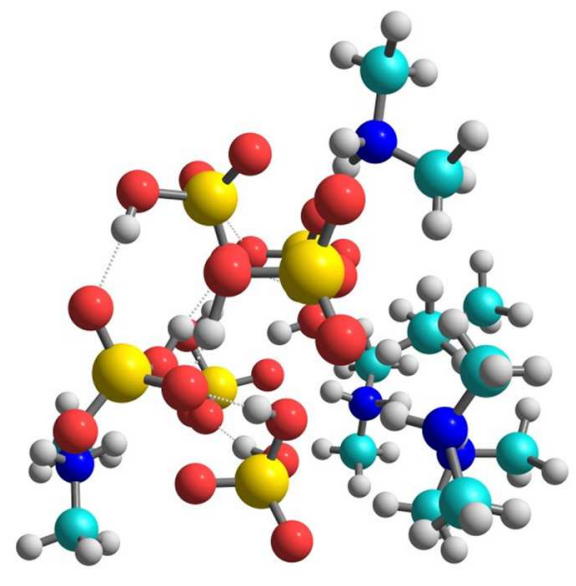

d

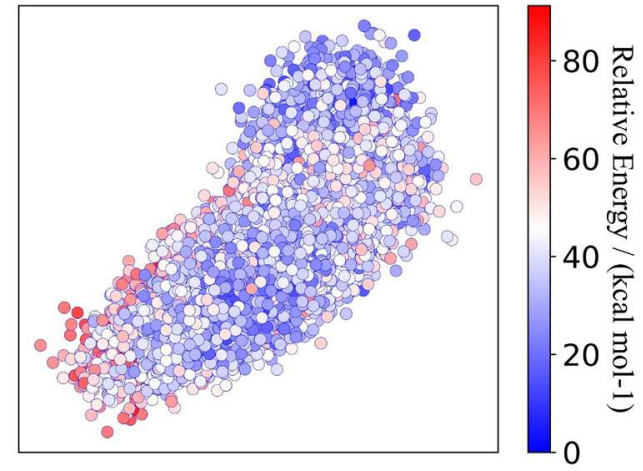

Figure 3 | Structural distribution for (SA)6(DMA)6 isomers derived from DNN-FF based MD. a shows the snapshots of MD for at $1 \mathrm{~ns}$. The cyan, white, red, blue and yellow circles represent $\mathrm{C}, \mathrm{H}, \mathrm{O}, \mathrm{N}$ and $\mathrm{S}$ atoms respectively. The $\mathrm{N}$ and $\mathrm{S}$ atoms radii are enlarged three times for clarity. b, The most stable isomer in trajectory. $\mathbf{c}$, Radial distribution function (RDF) between cluster center of mass (COM) and the five elements. d, Kernel principal component analysis (KPCA) ${ }^{20}$ maps of isomers using a global Smooth Overlap of Atomic Positions (SOAP) ${ }^{21}$ kernel.

Structural and energetic characteristics from DNN-FF based MD. With robust size scalability of DNN model, nanosecond scale MD simulation for cluster collision and evaporation can be made, whose representative snapshot is shown in Fig. 3a. Furthermore, the isolated cluster can be singled out for gaining insights about their structural evolution. Here (SA) 6 (DMA) 6 is chosen since it is the largest cluster with the same number of acids and bases observed in DNN-FF based MD, also very close to the lowest experimentally detectable size $(\sim 1.7 \mathrm{~nm})^{5}$. Surprisingly, three instead of all dimethylamine molecules are pronated initially (This is why only nine H-N distances are plotted in Extended Data Figure 5). For the most stable isomer (Fig. 3b), the sulfuric acid molecules are bonded with each other through hydrogen bonds, formed a shell with the cluster center of mass (COM) inside. The phenomena that the hydrogen bonded sulfuric acid molecules forms the first shell is general across all the (SA) $)_{6}(\mathrm{DMA})_{6}$ isomers as shown in the radial distribution function (RDF) from Fig. 3c. The more inner position of COM-S and COM-O than that of COM-N highlights the feature of the first shell composing of sulfuric acid molecules. The structural similarity can also 
be seen through the closely connected points in the energy basin (Fig. 3d). Besides, here $(\mathrm{SA})_{6}(\mathrm{DMA})_{6}$ cluster is from the collision between $(\mathrm{SA})_{5}(\mathrm{DMA})_{5}$ and $(\mathrm{SA})_{1}(\mathrm{DMA})_{1}$ and the high energy isomers during colliding and subsequently rearrangements can also be seen in Fig. $3 \mathrm{~d}$ (semitransparent red points in the lower left corner). Interestingly, one proton transfer event among dimethylamine molecules occurs at around 963 ps (Extended Data Figure 5). Comparatively, tons of proton transfer events occur among sulfuric acid molecules, making the proton number within one sulfuric acid molecules jumping from zero to three (Extended Data Figure 6). Notably, although sulfuric acid without protons or with three protons is rarely seen, we see several events whose duration is more than 100 fs. From above analysis, essential structural insights can be obtained through collecting the clusters with the same composition from MD trajectory.

\section{a}

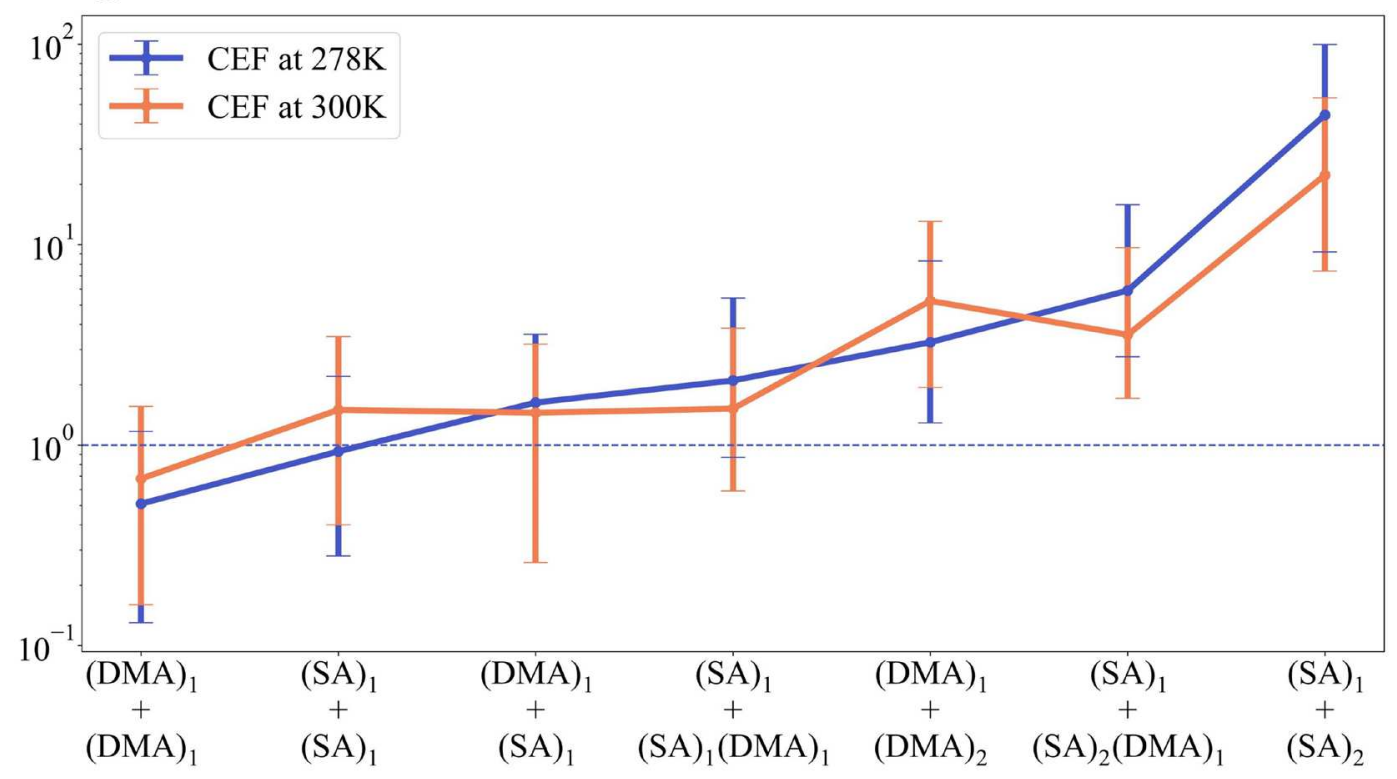

b
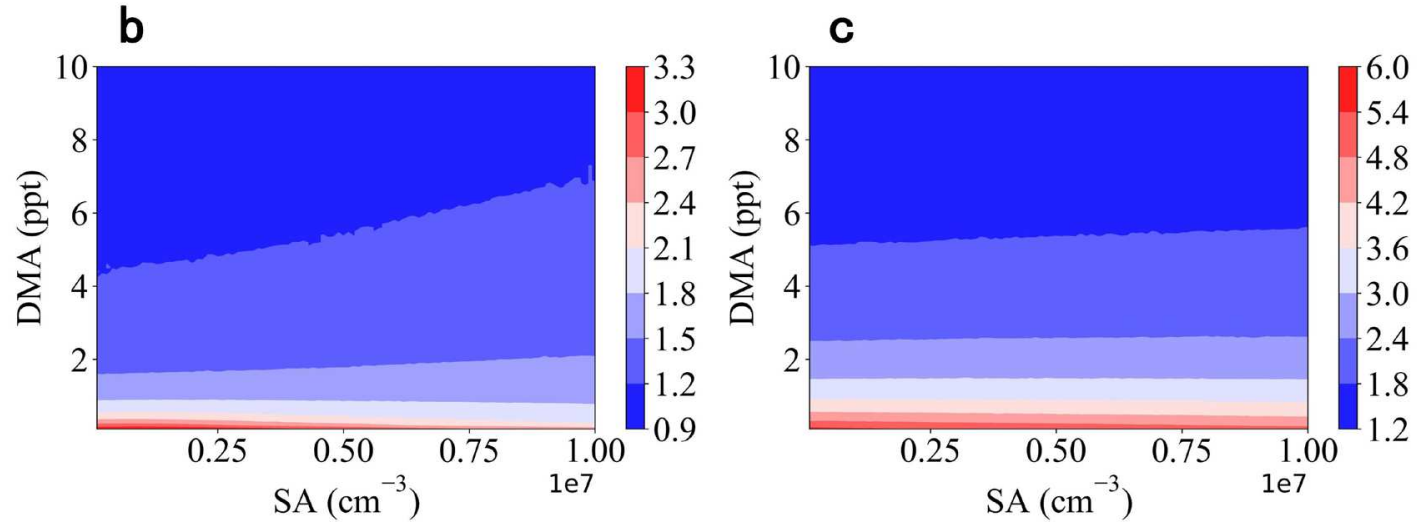

Figure 4 | Collision and Formation Rate Enhancement Factor (CEF and FREF). a, The collision and temperature dependence of CEF. Here only reactions both appearing at $278 \mathrm{~K}$ and 300 $\mathrm{K}$ are shown, the full reaction list can be found in Extended Data Table 2. $\mathbf{b}$ and $\mathbf{c}$ give the FREF for representative clean $\left(\mathrm{T}=278 \mathrm{~K}, \mathrm{CS}=2.6 \times 10^{-3} \mathrm{~s}^{-1}\right)$ and polluted environment $(\mathrm{T}=278 \mathrm{~K}, \mathrm{CS}=2.7$ $\left.\times 10^{-2} \mathrm{~s}^{-1}\right)$ respectively.

Aerosol nucleation kinetics. DNN-FF driven MD simulation of molecular clusters collisions and evaporations follow Poisson distribution ${ }^{22}$, so that the so-called Collision Enhancement Factor (CEF) 
can be derived. It is the quotient of MD derived collision rate constants divided by hard spheres collision rate constants. CEF is typically more than one due to the long-range intermolecular forces ${ }^{14}$. However, for the collision between dimethylamine monomer, CEF is below one mainly because of the intermolecular repulsion of dimethylamine molecules (Fig. 4a). To be noted, considering that long-range interaction in DNN is limited within the cutoff, the CEF here is certainly underestimated. Despite this, this shortcoming doesn't affect the validity and application value of the framework. The temperature dependence of $\mathrm{CEF}$ is not strong and not clearly distinguished comparing that from $278 \mathrm{~K}$ with $300 \mathrm{~K}$. Moreover, the composition dependence of CEF is also not clear, but for most collisions, $\mathrm{CEF}$ is more than one. Replacing hard spheres collision rate constants with MD derived ones in cluster kinetics model paves the way for fully ab initio simulation of aerosol nucleation. The derived formation rates divided by those based hard spheres collision rate constants give FREF (Fig. $4 \mathrm{~b}$ and $4 \mathrm{c}$ ). In representative clean (Fig.4b) and polluted (Fig. 4c) environment, FREF has strong negative correlation with DMA concentration and weak negative correlation with SA concentration. Under the same nucleation precursor concentration, FREF in polluted environments is larger than that in clean environment. Considering typically less precursor concentrations in clean environment than those in polluted environment, which one case has the larger FREF depends on the specific precursor concentrations. Generally speaking, here FREF ranges from one to six or so, however, this is the lower bound values as still there are quite a number of collision rate constants needing to be replaced and the underestimation of $\mathrm{CEF}$ due to lack of electrostatic interaction beyond the cutoff in DNN-FF. So the larger formation rates than expected before challenges the idea that SA-DMA nucleation is collision-limited with zero evaporation rates $^{23}$, providing the alternative scenario that collision rate constants are underestimated while evaporation rates are low but not zero. Further studies about this are definitely needed when fully ab initio kinetics are available in the future.

\section{Discussion}

Currently the coupling between machine learning (ML) and chemistry is on the rise, so training a DNN model with good performance on train and test data sets are becoming more and more routine ${ }^{24-32}$. However, training a DNN model with good size scalability and applicable on reactive MD simulation, especially for flexible molecules in this case is still very challenging ${ }^{33}$. Robust MD simulations here prove the high quality of data set given by passive learning and active learning as well as the excellent performance of descriptors combined with neural network framework and parameters.

Further directions lie in how to produce compact data set first, probably heavily relying on active learning. Another aspect is to improve the DNN model accuracy, possibly through transfer learning ${ }^{34}$ or $\Delta$-learning ${ }^{35}$. For nucleation applications, more diverse box size and initial monomer spatial distribution MD simulations need to be conducted to lower the uncertainties ${ }^{22}$ to estimate collision rate constants. Besides, long-range interaction beyond the cutoff size ( $6 \AA$ here) should be carefully considered in addition that larger box size simulation with more molecules inside is needed to include more various collisions, so that the simulation could be fully ab initio. Afterwards, the lots of $a b$ initio derived collision rates could tentatively be predicted purely by molecular physical chemistry properties to save simulation time costs.

The complex and various nucleation precursors in ambient environment, especially in polluted environment, call for new theoretical methods in addition to static QC to unravel the complication mechanisms. We believe the framework proposed here that the introducing of DNN-FF and the bridging between MD derived rate constants with cluster kinetics paves towards the fully ab initio 
simulation of atmosphere aerosol nucleation. The high accurate formation rate derived here can be further parametrized into climate model to improve the climate prediction in a global and local scale.

\section{Methods}

Passive learning. Instead of sampled by Basin-Hopping ${ }^{36}$ that is already widely applied in atmospheric non-covalent interaction clusters $^{37}$, the nucleation clusters PES is sampled by metadynamics (MetaMD $)^{38}$ due to its ability to sample high energy isomers, to prepare the initial data set for further active learning. The bump perturbation can be calculated as

$$
V_{\text {bump }}(\vec{R})=\sum_{\alpha} \lambda e^{-\sum_{i j} \frac{D_{i j}(\vec{R})-D_{i j}^{\alpha}}{2 \sigma^{2}}}
$$

Here $\alpha$ sums over snapshots of geometries where a "bump" has been placed at a particular value of the collective variable (CV), and $D_{i j}$ is the (possibly sparse) atomic distance matrix at a given point during the trajectory. The MD temperature, bump width $\sigma$ and bump height $\lambda$ is set to be $600 \mathrm{~K}, 2.0$ and 1.0 respectively. Clusters whose size is within the range of $(\mathrm{SA})_{\mathrm{m}}(\mathrm{DMA})_{\mathrm{n}}(\mathrm{m}=0-4, \mathrm{n}=0-4)$ is sampled with MetaMD coupled with PM7 semi-empirical method in Gaussian $16^{39}$. To be noted, to save computational costs, not all sizes are sampled. According to the experimental and theoretical predictions, SA-DMA systems tend to grow with the similar molecules number within cluster ${ }^{5}$, so for relatively larger cluster in the box, clusters with equal number of acid and base molecules or one more or less than the other are included. Mostly 50000 structures are sampled and then selected by Farthest Point Sampling (FPS) based on Many-body Tensor Representation (MBTR) descriptor ${ }^{40}$ for further DFT $(\omega \mathrm{B} 97 \mathrm{XD} / 6-31++\mathrm{G}(\mathrm{d}, \mathrm{p}))$ force calculations to be labelled. Systematic benchmark ${ }^{41}$ for aerosol nucleation clusters proves the good balance between accuracy and cost for $\omega \mathrm{B} 97 \mathrm{XD} / 6$ $31++\mathrm{G}(\mathrm{d}, \mathrm{p})$. The detailed MetaMD sampling and subsequent DFT calculations procedures are listed in Extended Data Table 3.

Active learning. Based on the initial data set prepared by MetaMD, active learning or on-the-fly strategy ${ }^{42}$ is utilized. The MetaMD sampling subset after screening is the initial data set to kick off the active learning iterations. In each iteration, firstly, 400 thousands steps training with different seeds is conducted to generate four DNN models. Then, NVT ensemble MD simulation is made based on trained DNN models. During the MD, four DNN models are utilized to pinpoint the candidate cluster whose error indicator satisfies the threshold range. The error indicator is the maximal standard deviation of the atomic force predicted by the model ensemble. The threshold ranges from $0.35 \mathrm{eV} / \AA$ to $0.50 \mathrm{eV} / \AA$, indicating that those whose error indicator is below $0.35 \mathrm{eV} / \AA$ are regarded as accurate and those whose error indicator is above $0.50 \mathrm{eV} / \AA$ are regarded as physically unreasonable. Finally, the energies and forces of candidate clusters are calculated through $\omega \mathrm{B} 97 \mathrm{XD} / 6-31++\mathrm{G}(\mathrm{d}, \mathrm{p})$ in Gaussian $16^{39}$ package and then put into the data set for training in the next iteration. To be noted, candidate clusters are carved out from the frame according to the interatomic distance cutoff, $3.5 \AA$. Different from the similar work conducted for combustion reactions ${ }^{43}$, here the cluster is obtained as long as the shortest interatomic distance between molecules is short than cutoff value in order to keep integrity of molecular cluster. The detailed iteration processes are listed in Extended Data Table 4.

DNN model. The smooth version of deep potential ${ }^{44,45}$ model is conducted here. In deep potential, the potential energy of molecular cluster is a sum of "atomic energies" $E=\sum_{i} E_{i}$, where $E_{i}$ is 
determined by the local environment of atom $i$ within a cutoff radius. The model includes two networks: the embedding network and the fitting network. The embedding network is of size (25, $50,100)$ and the fitting network is of size $(240,240,240)$. The fitting network uses ResNet architecture $^{46}$. The cutoff radius was set to $6.0 \AA$ and the descriptors decay smoothly from 5.8 to 6.0 $\AA$. The learning rate starts at $1.0 \times 10^{-3}$ and exponentially decays every 2,000 steps in 400,000 training steps in each active learning iteration and exponentially decays every 20,000 steps in $4,000,000$ training steps based on the final data set. The loss function is defined as a sum of different mean square errors of the DNN predictions for energy and force.

Molecular dynamics. Molecular cluster collision and evaporation simulations are conducted under NVT ensemble through LAMMPS ${ }^{47}$ package with 10 SA molecules and 10 DMA molecules initially randomly placing in the cubic box with length of $100 \AA$. The random positions are given by the aid of packmol ${ }^{48}$ package with the stable monomer of SA and DMS as the input. The temperature is kept at $278 \mathrm{~K}$ for 17 cases and $300 \mathrm{~K}$ for 16 cases respectively. In each case, MD is made firstly for 100 ps with the COM for each molecule being fixed for equilibration and then 1 ns for production. The positions under each frame within production stage are recorded every $10 \mathrm{fs}$ and the cluster index with the interatomic cutoff distance of $3.0 \AA$ is also recorded. The snapshot in Fig. 3a is plotted with $\mathrm{VMD}^{49}$ while the structure in Fig. $3 \mathrm{~b}$ is plotted with Chemcraft ${ }^{50}$. The structural clustering analysis in Fig. $3 \mathrm{~d}$ is conducted using ASAP ${ }^{51}$. The proton transfer distance threshold between $\mathrm{O}$ and $\mathrm{H}$ is set to $1.23 \AA$. Collison rate constants are derived according to the Poisson distribution feature of the reactive (collision or evaporation) events ${ }^{22}$ using the ChemTraYzer software package ${ }^{52}$. Collision and evaporation events are identified when duration is more than $100 \mathrm{fs}^{14}$. The Poissonbased collision rate constant $k$ can be calculated according to

$$
k=\frac{\sum_{j} N_{j}}{V \sum_{j}\left(\sum_{i}^{M} C_{i} \Delta t_{i}\right)_{j}}
$$

Here $N_{j}$ is the collision events number in case $j, V$ is the MD box volume $\left(1000 \mathrm{~nm}^{3}\right), i$ is the subsimulation number in case $j$ separated by reactive events, $C$ is the product of reactants concentration, $\Delta t$ is the interval between reactive events. The detailed derivation for rate constants and confidence interval of Poisson-based reaction events can be found in literature ${ }^{22}$.

Cluster kinetics. The molecular cluster kinetics simulations are made by our home-built python version $^{53}$ of Atmospheric Cluster Dynamics Code (ACDC) ${ }^{4}$ solving the ordinary differential equations. The collision rate constants are partially replaced by the MD observed collision events derived constants and the remains are calculated by hard sphere collision model. The evaporation rates are calculated assuming detailed balance based on quantum chemical thermodynamics ${ }^{11}$ from literature ${ }^{54}$. The condensation sink (CS) is set to be $2.6 \times 10^{-3} \mathrm{~s}^{-1}$ and $2.7 \times 10^{-2} \mathrm{~s}^{-1}$ to mimic condensation under clean ${ }^{55}$ and polluted ${ }^{56}$ environment respectively. In ACDC, the formation rate simulation can be calculated by

$$
J=\sum_{i=0}^{4} \sum_{j=0}^{4} \sum_{k=0}^{4} \sum_{l=0}^{4} \beta_{i k, j l} c_{i k} c_{j l}(i+j \geq 4, k+l>4)
$$

Here $i$ and $j$ refer to the number of SA molecules in the first and second cluster, $k$ and $l$ refer to the number of DMA molecules. The time evolution of cluster concentration $c_{i}$ can be obtained 
by solving the birth and death equations given by

$$
\frac{d_{c_{i}}}{d t}=\frac{1}{2} \sum_{j<i} \beta_{j,(i-j)} c_{j} c_{i-j}+\sum_{j} \gamma_{(i+j) \rightarrow i} c_{(i+j)}-\sum_{j} \beta_{i, j} c_{i} c_{j}-\frac{1}{2} \sum_{j<i} \gamma_{i \rightarrow j} c_{i}+C S
$$

Here CS represents condensation sink. $\beta_{i, j}$ represents the collision rate constants which obtained from hard sphere collision model and is calculated by

$$
\beta_{i, j}=\left(\frac{3}{4 \pi}\right)^{1 / 6}\left(\frac{6 k_{b} T}{m_{i}}+\frac{6 k_{b} T}{m_{j}}\right)^{1 / 2}\left(V_{i}^{1 / 3}+V_{j}^{1 / 3}\right)^{2}
$$

Here $T$ represents the temperature, $k_{b}$ represents the Boltzmann constant, and $m_{i}$ and $V_{i}$ represent the mass and volume of cluster $i$, respectively. The evaporation coefficient $\gamma_{(i+j) \rightarrow i}$ is calculated by

$$
\gamma_{(i+j) \rightarrow i, j}=\beta_{i, j} \frac{c_{i}^{e} c_{j}^{e}}{c_{i+j}^{e}}=\beta_{i, j} c_{\mathrm{ref}} \exp \left(\frac{\Delta G_{i+j}-\Delta G_{i}-\Delta G_{j}}{k_{b} T}\right)
$$

\section{References}

Frenkel, J. Statistical Theory of Condensation Phenomena. journal of chemical physics 7, 200201 (1939).

2 Vehkamäki, H. \& Riipinen, I. Thermodynamics and kinetics of atmospheric aerosol particle formation and growth. Chem Soc Rev 41, 5160-5173 (2012).

3 Merikanto, J., Zapadinsky, E., Lauri, A. \& Vehkamäki, H. Origin of the failure of classical nucleation theory: incorrect description of the smallest clusters. Phys Rev Lett 98, 145702 (2007).

4 McGrath, M. J. et al. Atmospheric Cluster Dynamics Code: a flexible method for solution of the birth-death equations. atmospheric chemistry and physics 12, 2345-2355 (2011).

5 Almeida, J. et al. Molecular understanding of sulphuric acid-amine particle nucleation in the atmosphere. Nature 502, 359-363 (2013)

6 Yao, L. et al. Atmospheric new particle formation from sulfuric acid and amines in a Chinese megacity. Science 361, 278-281 (2018).

7 Lehtipalo, K. et al. The effect of acid-base clustering and ions on the growth of atmospheric nano-particles. nature communications 7, 11594 (2016).

8 Liu, L. et al. Unexpected quenching effect on new particle formation from the atmospheric reaction of methanol with SO\&1t;sub\&gt;3\&1t;/sub\&gt. Proceedings of the National Academy of Sciences 116, 24966-24971 (2019).

9 Kumar, M., Li, H., Zhang, X., Zeng, X. C. \& Francisco, J. S. Nitric Acid-Amine Chemistry in the Gas Phase and at the Air-Water Interface. journal of the american chemical society $\mathbf{1 4 0}$, 6456-6466 (2018).

$10 \mathrm{Li}, \mathrm{H}$. et al. Self-Catalytic Reaction of $\mathrm{SO} 3$ and $\mathrm{NH} 3$ To Produce Sulfamic Acid and Its Implication to Atmospheric Particle Formation. J Am Chem Soc 140, 11020-11028 (2018).

Ortega, I. K. et al. From quantum chemical formation free energies to evaporation rates. atmospheric chemistry and physics 12, 225-235 (2011).

Elm, J. et al. Modeling the formation and growth of atmospheric molecular clusters: A review. 
J Aerosol Sci 149, 105621 (2020).

Kürten, A. et al. Neutral molecular cluster formation of sulfuric acid-dimethylamine observed in real time under atmospheric conditions. Proceedings of the National Academy of Sciences of the United States of America 111, 15019-15024 (2014).

Halonen, R., Zapadinsky, E., Kurtén, T., Vehkamäki, H. \& Reischl, B. Rate enhancement in collisions of sulfuric acid molecules due to long-range intermolecular forces. atmospheric chemistry and physics 19, 13355-13366 (2019).

15 Li, C. \& Signorell, R. Understanding vapor nucleation on the molecular level: A review. journal of aerosol science 153, 105676 (2021).

Salvalaglio, M., Tiwary, P., Maggioni, G. M., Mazzotti, M. \& Parrinello, M. Overcoming time scale and finite size limitations to compute nucleation rates from small scale well tempered metadynamics simulations. journal of chemical physics 145, 211925 (2016).

Stewart, J. J. P. Optimization of parameters for semiempirical methods VI: more modifications to the NDDO approximations and re-optimization of parameters. Journal of Molecular Modeling 19, 1-32 (2013).

Loukonen, V. et al. Enhancing effect of dimethylamine in sulfuric acid nucleation in the presence of water - a computational study. atmospheric chemistry and physics 10, 4961-4974 (2010).

19 Yue, S. et al. When do short-range atomistic machine-learning models fall short? journal of chemical physics 154, 034111 (2021).

Schölkopf, B., Smola, A. \& Müller, K.-R. Nonlinear component analysis as a kernel eigenvalue problem. Neural Computation 10, 1299-1319 (1998).

Bartók, A. P., Kondor, R. \& Csányi, G. On representing chemical environments. Phys. Rev. B 87, 184115 (2013).

Kröger, L. C., Kopp, W. A., Döntgen, M. \& Leonhard, K. Assessing Statistical Uncertainties of Rare Events in Reactive Molecular Dynamics Simulations. journal of chemical theory and computation 13, 3955-3960 (2017).

Kuerten, A. et al. New particle formation in the sulfuric acid-dimethylamine-water system: reevaluation of CLOUD chamber measurements and comparison to an aerosol nucleation and growth model. Atmospheric Chemistry and Physics 18, 845-863 (2018).

Dral, P. O. Quantum Chemistry in the Age of Machine Learning. J. Phys. Chem. Lett. 11, 23362347 (2020).

Noé, F., Tkatchenko, A., Müller, K. R. \& Clementi, C. Machine Learning for Molecular Simulation. annual review of physical chemistry 71, 361-390 (2020).

Mueller, T., Hernandez, A. \& Wang, C. Machine learning for interatomic potential models. journal of chemical physics 152, 50902 (2020).

Unke, O. T. et al. Machine Learning Force Fields. chemical reviews, DOI: 10.1021/acs.chemrev.1020c01111 (2021).

Behler, J. Four Generations of High-Dimensional Neural Network Potentials. chemical reviews, DOI: 10.1021/acs.chemrev.1020c00868 (2021).

Meuwly, M. Machine Learning for Chemical Reactions. chemical reviews, DOI: 10.1021/acs.chemrev.1021c00033 (2021).

30 Keith, J. A. et al. Combining Machine Learning and Computational Chemistry for Predictive Insights Into Chemical Systems. chemical reviews 121, 9816-9872 (2021). 

Phys. Chem. Lett. 12, 6227-6243 (2021).

32 Westermayr, J., Gastegger, M., Schütt, K. T. \& Maurer, R. J. Perspective on integrating machine learning into computational chemistry and materials science. The Journal of Chemical Physics 154, 230903 (2021).

Schran, C., Brieuc, F. \& Marx, D. Transferability of machine learning potentials: Protonated water neural network potential applied to the protonated water hexamer. journal of chemical physics 154, 051101 (2021).

Smith, J. S. et al. Approaching coupled cluster accuracy with a general-purpose neural network potential through transfer learning. nature communications 10, 2903 (2019).

Ramakrishnan, R., Dral, P. O., Rupp, M. \& Lilienfeld, O. A. v. Big Data Meets Quantum Chemistry Approximations: The $\Delta$-Machine Learning Approach. journal of chemical theory and computation 11, 2087-2096 (2015).

Jiang, S. et al. Study of $\mathrm{Cl}-(\mathrm{H} 2 \mathrm{O}) \mathrm{n}(\mathrm{n}=1-4)$ using basin - hopping method coupled with density functional theory. J Comput Chem 35, 159-165 (2014).

Zhang, J. \& Glezakou, V.-A. Global optimization of chemical cluster structures: Methods, applications, and challenges. Int J Quantum Chem 121, e26553 (2021).

Herr, J. E., Yao, K., McIntyre, R., Toth, D. W. \& Parkhill, J. Metadynamics for training neural network model chemistries: A competitive assessment. journal of chemical physics 148, 241710 (2018).

Huo, H. \& Rupp, M. J. a. p. a. Unified representation of molecules and crystals for machine learning. Preprint at https://arxiv.org/abs/1704.06439 (2017).

Elm, J. \& Mikkelsen, K. V. Computational approaches for efficiently modelling of small atmospheric clusters. Chem Phys Lett 615, 26-29 (2014).

Zhang, Y. et al. DP-GEN: A concurrent learning platform for the generation of reliable deep learning based potential energy models. computer physics communications 253, 107206 (2020).

Zeng, J., Cao, L., Xu, M., Zhu, T. \& Zhang, J. Z. H. Complex reaction processes in combustion unraveled by neural network-based molecular dynamics simulation. nature communications $\mathbf{1 1}$, 5713 (2020).

Wang, H., Zhang, L., Han, J. \& E, W. DeePMD-kit: A deep learning package for many-body potential energy representation and molecular dynamics. computer physics communications $\mathbf{2 2 8}$, 178-184 (2018).

Zhang, L., Han, J., Wang, H., Car, R. \& E, W. Deep Potential Molecular Dynamics: A Scalable Model with the Accuracy of Quantum Mechanics. physical review letters 120, 143001 (2018). He, K., Zhang, X., Ren, S. \& Sun, J. Deep Residual Learning for Image Recognition. In: Tuytelaars, T. et al. (eds) Proc. IEEE Conference on Computer Vision and Pattern Recognition (2016).

47 Aktulga, H. M., Fogarty, J. C., Pandit, S. A. \& Grama, A. Y. Parallel reactive molecular dynamics: Numerical methods and algorithmic techniques. Parallel Computing 38, 245-259 (2012).

Martínez, L., Andrade, R., Birgin, E. G. \& Martínez, J. M. PACKMOL: a package for building initial configurations for molecular dynamics simulations. journal of computational chemistry 30, 2157-2164 (2009). 
49 Humphrey, W., Dalke, A. \& Schulten, K. VMD: visual molecular dynamics. Journal of molecular graphics 14, 33-38 (1996).

50 Andrienko, G. J. U. w. h. w. c. c. Chemcraft - graphical software for visualization of quantum chemistry computations. https://www.chemcraftprog.com.

51 Cheng, B. et al. Mapping Materials and Molecules. accounts of chemical research 53, 19811991 (2020).

52 Döntgen, M. et al. Automated discovery of reaction pathways, rate constants, and transition states using reactive molecular dynamics simulations. Journal of chemical theory and computation 11, 2517-2524 (2015).

$53 \mathrm{Xu}, \mathrm{C} . \mathrm{X}$. et al. Formation of atmospheric molecular clusters of methanesulfonic acidDiethylamine complex and its atmospheric significance. Atmos Environ 226, 117404 (2020).

54 Olenius, T., Kupiainen-Määttä, O., Ortega, I. K., Kurtén, T. \& Vehkamäki, H. Free energy barrier in the growth of sulfuric acid-ammonia and sulfuric acid-dimethylamine clusters. The Journal of chemical physics 139, 084312 (2013).

55 Maso, M. D. et al. Annual and interannual variation in boreal forest aerosol particle number and volume concentration and their connection to particle formation. Tellus, Ser. B 60, 495-508 (2008).

56 Wu, Z. et al. New particle formation in Beijing, China: Statistical analysis of a 1 - year data set. J Geophys Res 112, D09209 (2007).

\section{Competing interests}

No potential conflicts of interest exist for any of the listed authors.

\section{Data and materials availability}

All data related to this study can be obtained from the corresponding author via email. 
Extended Data Table 1 | Root mean squared error (RMSE) of different method performance for describing the dimer detachment (in $\mathrm{kcal} / \mathrm{mol}$ ).

\begin{tabular}{|c|c|c|c|}
\hline & $(\mathrm{SA})_{2}$ & $(\mathrm{DMA})_{2}$ & $(\mathrm{SA})_{1}(\mathrm{DMA})_{1}$ \\
\hline PM7 & 1.74 & 1.51 & 14.92 \\
\hline DNN & 1.57 & 1.32 & 0.78 \\
\hline OPLS & 8.73 & Null & Null \\
\hline
\end{tabular}

Extended Data Table 2 | Collision rate constants $\left(\mathrm{cm}^{3} \mathrm{~s}^{-1}\right)$ from MD and hard sphere model at $278 \mathrm{~K}$ and $300 \mathrm{~K}$. Those constants' uncertain is below 2.0. $\mathrm{k}$, $\mathrm{k}_{\text {low }}$ and $\mathrm{k}_{\text {up }}$ represent the average, upper and lower rate constants from MD while $\mathrm{k}_{\mathrm{gas}}$ represent those from hard sphere model.

a) $\mathrm{T}=278 \mathrm{~K}$ :

\begin{tabular}{|c|c|c|c|c|c|c|c|}
\hline reaction & $\mathrm{k}$ & $\mathrm{k}_{\text {low }}$ & $\mathrm{k}_{\text {up }}$ & $\mathrm{k} / \mathrm{k}_{\text {low }}$ & $\mathrm{k}_{\text {up }} / \mathrm{k}$ & $\mathrm{k}_{\text {gas }}$ & $\mathrm{k} / \mathrm{k}_{\text {gas }}$ \\
\hline$(\mathrm{DMA})_{1}+(\mathrm{DMA})_{1}->(\mathrm{DMA})_{2}$ & $2.88 \mathrm{e}-10$ & $2.15 \mathrm{e}-10$ & $3.75 \mathrm{e}-10$ & 1.34 & 1.30 & $5.68 \mathrm{e}-10$ & 0.51 \\
\hline$(\mathrm{SA})_{1}+(\mathrm{SA})_{1}->(\mathrm{SA})_{2}$ & $3.10 \mathrm{e}-10$ & $2.17 \mathrm{e}-10$ & $4.26 \mathrm{e}-10$ & 1.43 & 1.38 & $3.34 \mathrm{e}-10$ & 0.93 \\
\hline$(\mathrm{DMA})_{1}+(\mathrm{SA})_{1}->(\mathrm{SA})_{1}(\mathrm{DMA})_{1}$ & $7.39 \mathrm{e}-10$ & $6.18 \mathrm{e}-10$ & $8.76 \mathrm{e}-10$ & 1.20 & 1.19 & $4.53 \mathrm{e}-10$ & 1.63 \\
\hline$(\mathrm{SA})_{1}+(\mathrm{SA})_{1}(\mathrm{DMA})_{1}->(\mathrm{SA})_{2}(\mathrm{DMA})_{1}$ & $8.59 \mathrm{e}-10$ & $5.02 \mathrm{e}-10$ & $1.35 \mathrm{e}-09$ & 1.71 & 1.58 & $4.08 \mathrm{e}-10$ & 2.10 \\
\hline$(\mathrm{DMA})_{1}+(\mathrm{DMA})_{2}->(\mathrm{DMA})_{3}$ & $2.05 \mathrm{e}-09$ & $1.24 \mathrm{e}-09$ & $3.16 \mathrm{e}-09$ & 1.65 & 1.54 & $6.28 \mathrm{e}-10$ & 3.26 \\
\hline$(\mathrm{SA})_{1}+(\mathrm{SA})_{2}(\mathrm{DMA})_{1}->(\mathrm{SA})_{3}(\mathrm{DMA})_{1}$ & $2.54 \mathrm{e}-09$ & $1.35 \mathrm{e}-09$ & $4.27 \mathrm{e}-09$ & 1.88 & 1.68 & $4.31 \mathrm{e}-10$ & 5.90 \\
\hline$(\mathrm{SA})_{1}+(\mathrm{SA})_{2}->(\mathrm{SA})_{3}$ & $1.64 \mathrm{e}-08$ & $1.30 \mathrm{e}-08$ & $2.04 \mathrm{e}-08$ & 1.26 & 1.24 & $3.69 \mathrm{e}-10$ & 44.39 \\
\hline
\end{tabular}

b) $\mathrm{T}=300 \mathrm{~K}$ :

\begin{tabular}{|c|c|c|c|c|c|c|c|}
\hline reaction & $\mathrm{k}$ & $\mathrm{k}_{\text {low }}$ & $\mathrm{k}_{\text {up }}$ & $\mathrm{k} / \mathrm{k}_{\text {low }}$ & $\mathrm{k}_{\text {up }} / \mathrm{k}$ & $\mathrm{k}_{\text {gas }}$ & $\mathrm{k} / \mathrm{k}_{\text {gas }}$ \\
\hline$(\mathrm{DMA})_{1}+(\mathrm{DMA})_{1}->(\mathrm{DMA})_{2}$ & $4.04 \mathrm{e}-10$ & $3.07 \mathrm{e}-10$ & $5.20 \mathrm{e}-10$ & 1.32 & 1.29 & $5.90 \mathrm{e}-10$ & 0.68 \\
\hline$(\mathrm{DMA})_{1}+(\mathrm{SA})_{1}(\mathrm{DMA})_{1}->(\mathrm{SA})_{1}(\mathrm{DMA})_{2}$ & $5.82 \mathrm{e}-10$ & $3.71 \mathrm{e}-10$ & $8.61 \mathrm{e}-10$ & 1.57 & 1.48 & $5.89 \mathrm{e}-10$ & 0.99 \\
\hline$(\mathrm{DMA})_{1}+(\mathrm{SA})_{1}->(\mathrm{SA})_{1}(\mathrm{DMA})_{1}$ & $6.81 \mathrm{e}-10$ & $5.58 \mathrm{e}-10$ & $8.20 \mathrm{e}-10$ & 1.22 & 1.20 & $4.70 \mathrm{e}-10$ & 1.45 \\
\hline$(\mathrm{SA})_{1}+(\mathrm{SA})_{1}->(\mathrm{SA})_{2}$ & $5.19 \mathrm{e}-10$ & $3.81 \mathrm{e}-10$ & $6.86 \mathrm{e}-10$ & 1.36 & 1.32 & $3.47 \mathrm{e}-10$ & 1.50 \\
\hline$(\mathrm{SA})_{1}+(\mathrm{SA})_{1}(\mathrm{DMA})_{1}->(\mathrm{SA})_{2}(\mathrm{DMA})_{1}$ & $6.43 \mathrm{e}-10$ & $3.95 \mathrm{e}-10$ & $9.79 \mathrm{e}-10$ & 1.63 & 1.52 & $4.24 \mathrm{e}-10$ & 1.52 \\
\hline$(\mathrm{SA})_{1}+(\mathrm{SA})_{2}(\mathrm{DMA})_{1}->(\mathrm{SA})_{3}(\mathrm{DMA})_{1}$ & $1.59 \mathrm{e}-09$ & $8.21 \mathrm{e}-10$ & $2.73 \mathrm{e}-09$ & 1.94 & 1.72 & $4.47 \mathrm{e}-10$ & 3.55 \\
\hline$(\mathrm{DMA})_{1}+(\mathrm{SA})_{2}->(\mathrm{SA})_{2}(\mathrm{DMA})_{1}$ & $2.20 \mathrm{e}-09$ & $1.37 \mathrm{e}-09$ & $3.31 \mathrm{e}-09$ & 1.61 & 1.51 & $5.46 \mathrm{e}-10$ & 4.03 \\
\hline$(\mathrm{DMA})_{1}+(\mathrm{DMA})_{2}->(\mathrm{DMA})_{3}$ & $3.42 \mathrm{e}-09$ & $2.15 \mathrm{e}-09$ & $5.10 \mathrm{e}-09$ & 1.59 & 1.49 & $6.52 \mathrm{e}-10$ & 5.24 \\
\hline$(\mathrm{DMA})_{1}+(\mathrm{SA})_{3}(\mathrm{DMA})_{1}->(\mathrm{SA})_{3}(\mathrm{DMA})_{2}$ & $1.32 \mathrm{e}-08$ & $7.22 \mathrm{e}-09$ & $1.45 \mathrm{e}-08$ & 1.82 & 1.10 & $6.98 \mathrm{e}-10$ & 18.91 \\
\hline$(\mathrm{SA})_{1}+(\mathrm{SA})_{2}->(\mathrm{SA})_{3}$ & $8.53 \mathrm{e}-09$ & $5.70 \mathrm{e}-09$ & $1.22 \mathrm{e}-08$ & 1.50 & 1.43 & $3.84 \mathrm{e}-10$ & 22.23 \\
\hline$(\mathrm{SA})_{1}+(\mathrm{SA})_{4}(\mathrm{DMA})_{1}->(\mathrm{SA})_{5}(\mathrm{DMA})_{1}$ & $2.12 \mathrm{e}-08$ & $1.09 \mathrm{e}-08$ & $3.64 \mathrm{e}-08$ & 1.94 & 1.72 & $5.08 \mathrm{e}-10$ & 41.70 \\
\hline
\end{tabular}

Extended Data Table 3 | Passive learning and active learning sampling structure number for the corresponding cluster composition.

\begin{tabular}{|c|c|c|c|}
\hline \multirow{2}{*}{$\begin{array}{c}\text { Cluster } \\
\text { composition }\end{array}$} & \multicolumn{2}{|c|}{ Passive learning } & Active learning \\
\cline { 2 - 4 } & Metadynamics sampling & DFT calculations & DFT calculations \\
\hline$(\text { DMA })_{1}$ & 99999 & 69678 & 2 \\
\hline$(\text { DMA })_{2}$ & 50000 & 15536 & 22 \\
\hline$(\text { DMA })_{3}$ & 100000 & 4998 & 2 \\
\hline$(D M A)_{4}$ & 0 & 0 & 13 \\
\hline$(S A)_{1}$ & 257973 & 58872 & 2 \\
\hline$(\mathrm{SA})_{1}(\mathrm{DMA})_{1}$ & 120000 & 25171 & 84 \\
\hline
\end{tabular}




\begin{tabular}{|c|c|c|c|}
\hline$(\mathrm{SA})_{1}(\mathrm{DMA})_{2}$ & 50000 & 10532 & 447 \\
\hline$(\mathrm{SA})_{1}(\mathrm{DMA})_{3}$ & 0 & 0 & 54 \\
\hline$(\mathrm{SA})_{1}(\mathrm{DMA})_{4}$ & 0 & 0 & 57 \\
\hline$(\mathrm{SA})_{2}$ & 144320 & 4610 & 51 \\
\hline$(\mathrm{SA})_{2}(\mathrm{DMA})_{1}$ & 49999 & 16377 & 63 \\
\hline$(\mathrm{SA})_{2}(\mathrm{DMA})_{2}$ & 50000 & 4339 & 106 \\
\hline$(\mathrm{SA})_{2}(\mathrm{DMA})_{3}$ & 50000 & 4998 & 13 \\
\hline$(\mathrm{SA})_{2}(\mathrm{DMA})_{4}$ & 0 & 0 & 201 \\
\hline$(\mathrm{SA})_{2}(\mathrm{DMA})_{5}$ & 0 & 0 & 14 \\
\hline$(\mathrm{SA})_{3}$ & 50303 & 5028 & 24 \\
\hline$(\mathrm{SA})_{3}(\mathrm{DMA})_{1}$ & 0 & 0 & 3 \\
\hline$(\mathrm{SA})_{3}(\mathrm{DMA})_{2}$ & 49863 & 4801 & 62 \\
\hline$(\mathrm{SA})_{3}(\mathrm{DMA})_{3}$ & 49998 & 4992 & 51 \\
\hline$(\mathrm{SA})_{3}(\mathrm{DMA})_{4}$ & 49997 & 4987 & 12 \\
\hline$(\mathrm{SA})_{4}$ & 50000 & 4014 & 0 \\
\hline$(\mathrm{SA})_{4}(\mathrm{DMA})_{3}$ & 49997 & 4991 & 146 \\
\hline$(\mathrm{SA})_{4}(\mathrm{DMA})_{4}$ & 49867 & 2315 & 0 \\
\hline$(\mathrm{SA})_{4}(\mathrm{DMA})_{5}$ & 50293 & 200 & 17 \\
\hline$(\mathrm{SA})_{5}(\mathrm{DMA})_{4}$ & 49999 & 200 & 0 \\
\hline$(\mathrm{SA})_{5}(\mathrm{DMA})_{5}$ & 50000 & 200 & 109 \\
\hline$(\mathrm{SA})_{5}(\mathrm{DMA})_{6}$ & 49999 & 191 & 0 \\
\hline Total & 1522607 & 247030 & 1555 \\
\hline $\begin{array}{l}\text { Percentage of } \\
\text { total DFT } \\
\text { structure number }\end{array}$ & Null & $99.37 \%$ & $0.63 \%$ \\
\hline
\end{tabular}

Extended Data Table $4 \mid$ Molecular dynamics parameters in active learning iterations.

\begin{tabular}{|c|c|c|c|c|}
\hline $\begin{array}{l}\text { Iteration } \\
\text { number }\end{array}$ & $\begin{array}{l}\text { Initial molecular } \\
\text { composition }^{\mathrm{a}}\end{array}$ & box length & length(ps) & structures number \\
\hline 0 & \multirow{6}{*}{$(4,4)$} & \multirow{6}{*}{40} & 2 & 13 \\
\hline 1 & & & 2 & 27 \\
\hline 2 & & & 10 & 36 \\
\hline 3 & & & 10 & 103 \\
\hline 4 & & & 20 & 89 \\
\hline 5 & & & 50 & 172 \\
\hline 6 & \multirow{3}{*}{$(5,5)$} & \multirow{3}{*}{50} & 100 & 168 \\
\hline 7 & & & 200 & 480 \\
\hline 8 & & & 500 & 467 \\
\hline
\end{tabular}

Notes: a. $(m, n)$ represents the number of SA and DMA molecules respectively. $b$. structures number gives the total cluster structures number derived from each active learning iteration. 
Extended Data Figure 1 | Formation of (SA) ${ }_{6}(\mathrm{DMA})_{6}$ in DNN-MD arising from the collision of $(\text { SA })_{1}(\text { DMA })_{1}$ and (SA $)_{5}(\text { DMA })_{5}$.

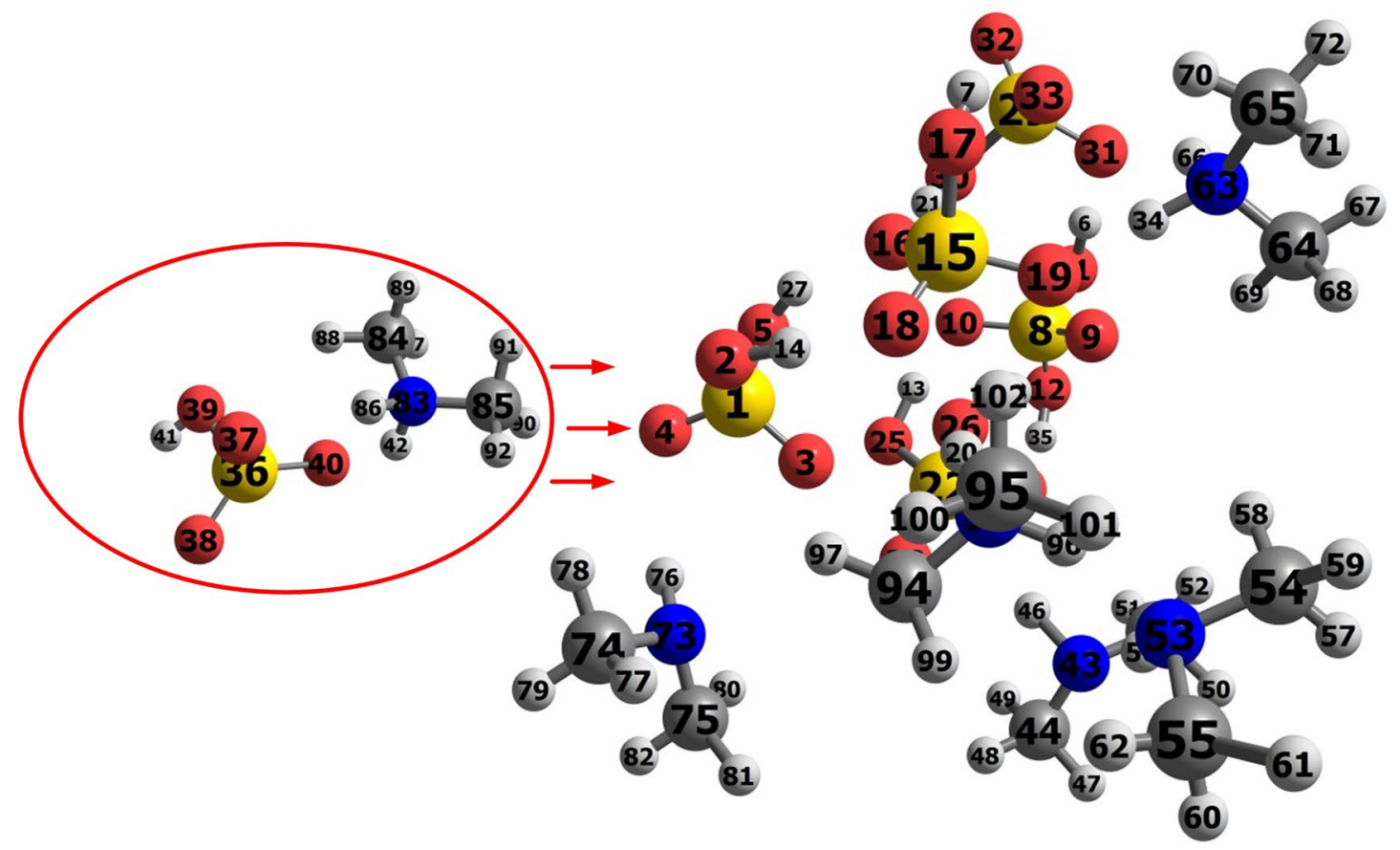

Extended Data Figure 2 | Cluster shape anisotropy for each composition with uncertain bar at $278 \mathrm{~K}$.

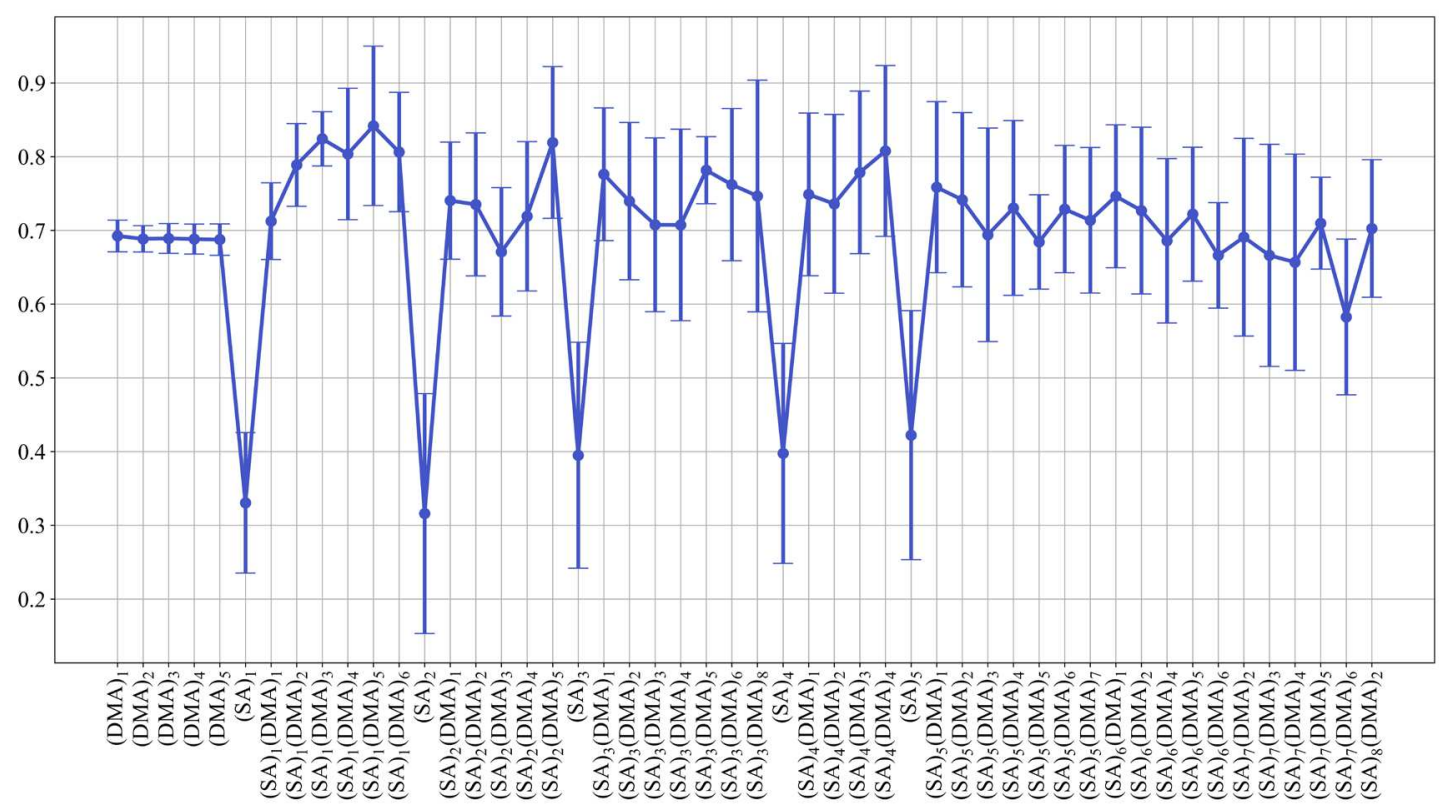


Extended Data Figure 3 | Cluster shape anisotropy for each composition with uncertain bar at $300 \mathrm{~K}$.

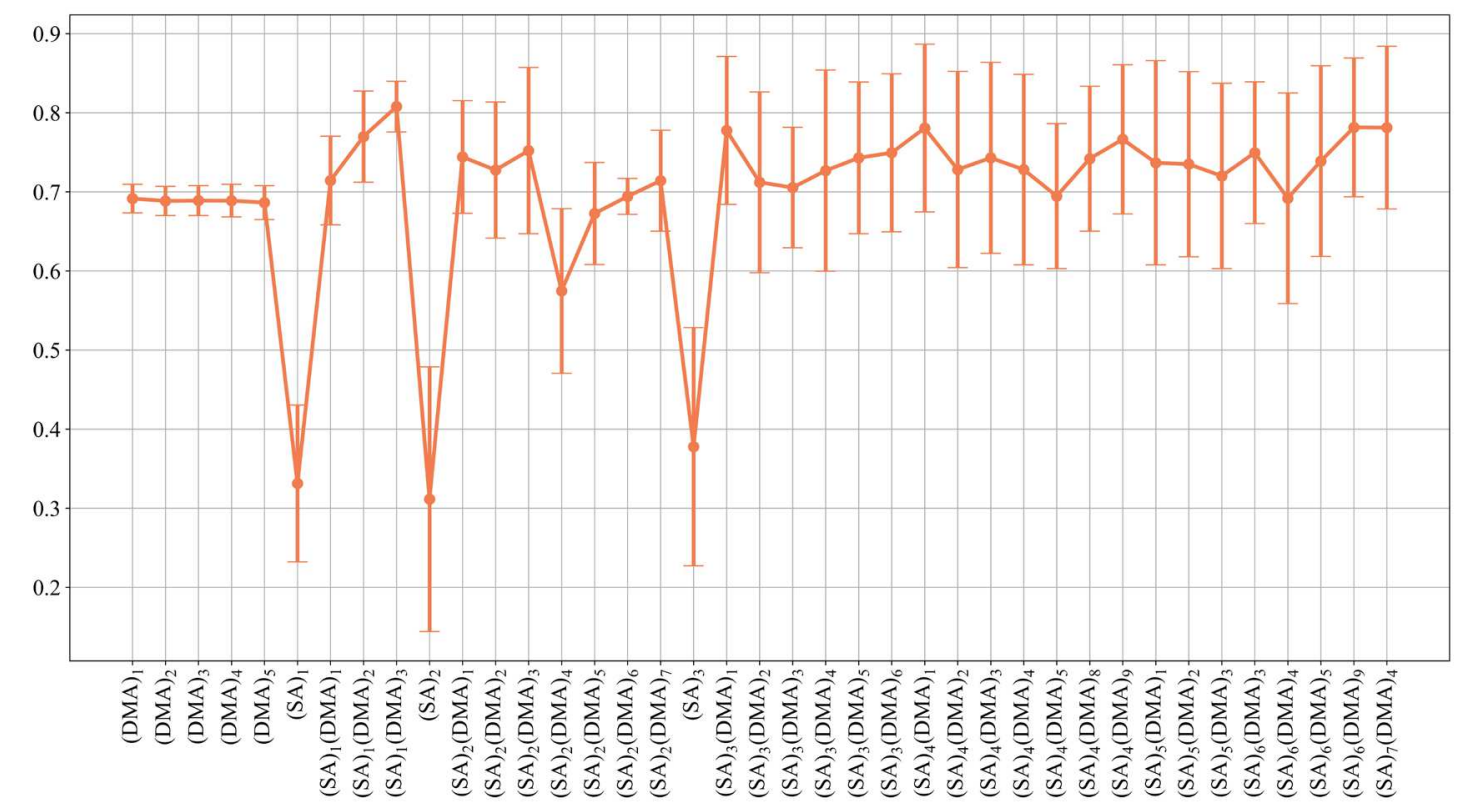

Extended Data Figure $4 \mid \mathrm{H}$ and $\mathrm{O}$ atom distance for each sulfuric acid in (SA)6(DMA)6 6 cluster. Nine H-O pairs with covalent bonds in sulfuric acid molecules observed in the initial moment of $(\mathrm{SA})_{6}(\mathrm{DMA})_{6}$ formation are plotted below.

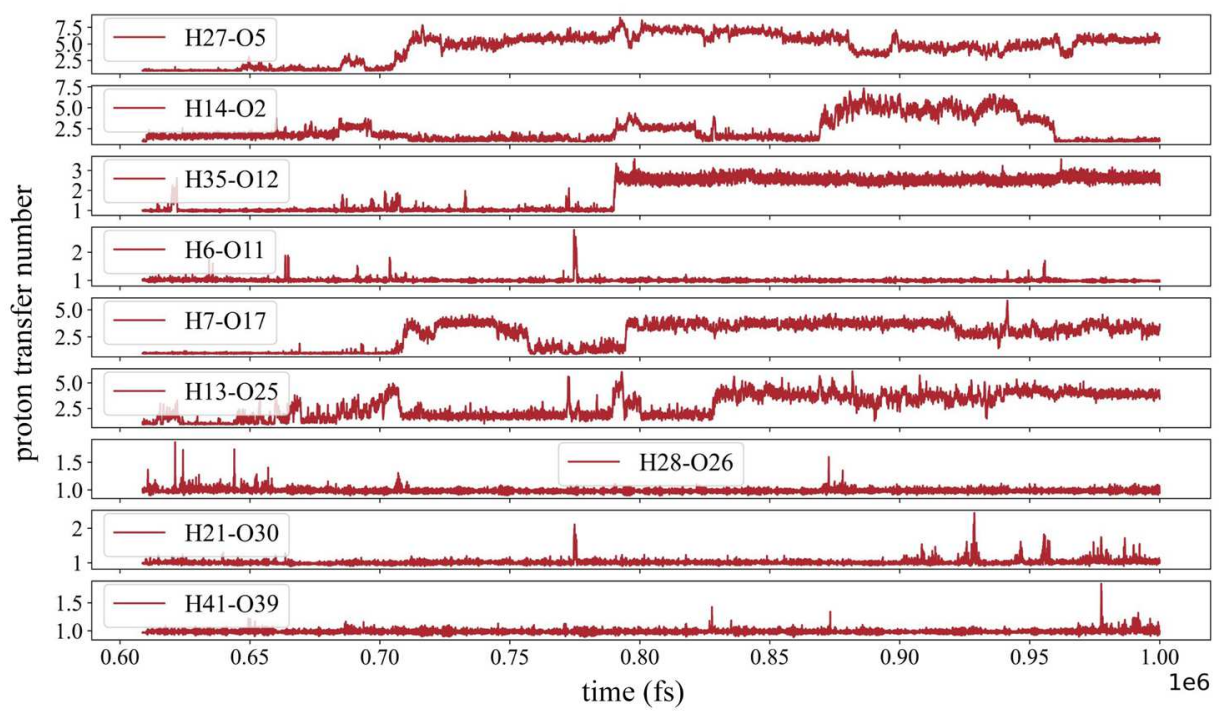


Extended Data Figure 5 | Time evolution of nine hydrogen nitrogen pairs distance for (SA)6(DMA) 6 cluster.

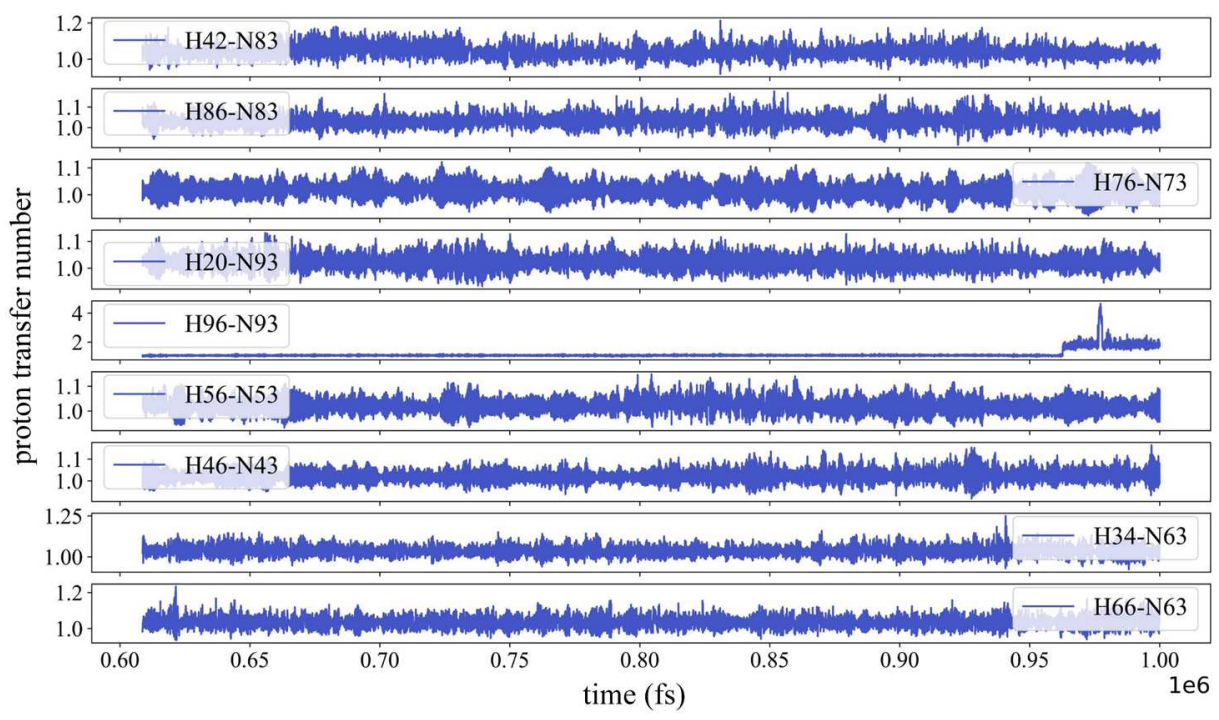

Extended Data Figure 6 | Time evolution of proton number within each sulfuric acid molecule for (SA) 6 (DMA) 6 cluster. To be noted, the time evolution is filtered with the proton number duration being more than 100 fs for observing relatively long existence of bonds.

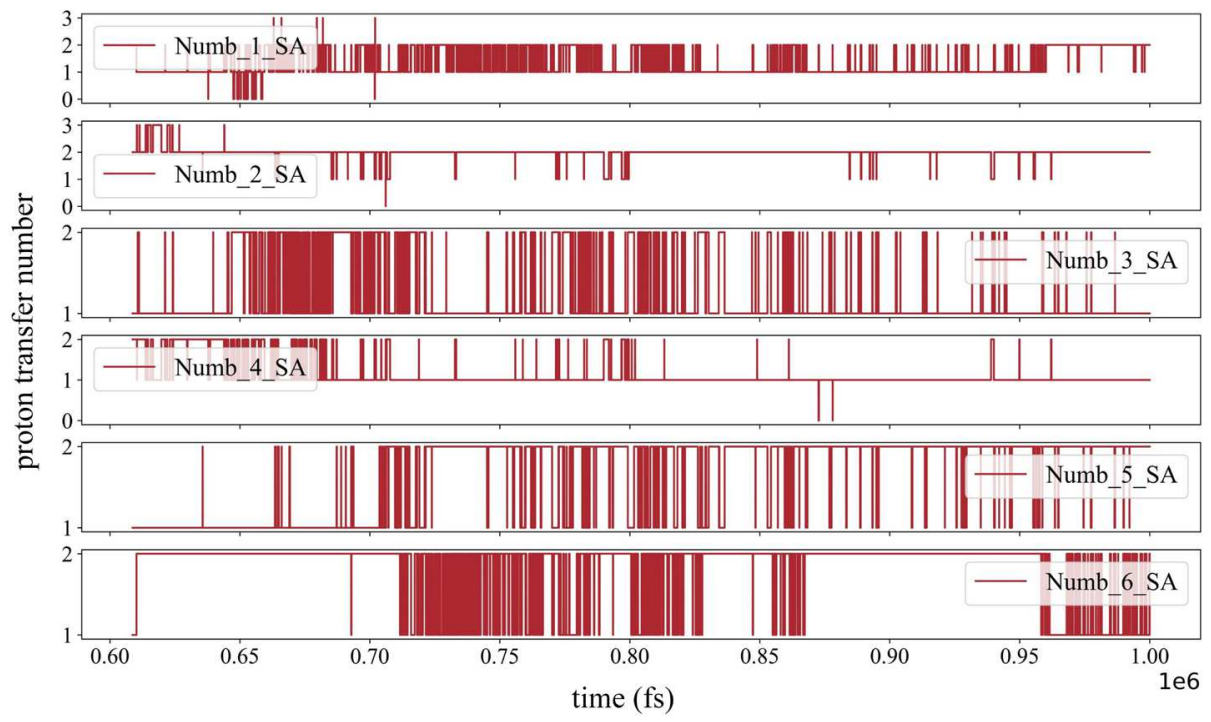


Extended Data Figure 7 | Radial distribution function (RDF) for clusters with the same number of acid and base molecules inside at $278 \mathrm{~K}$.
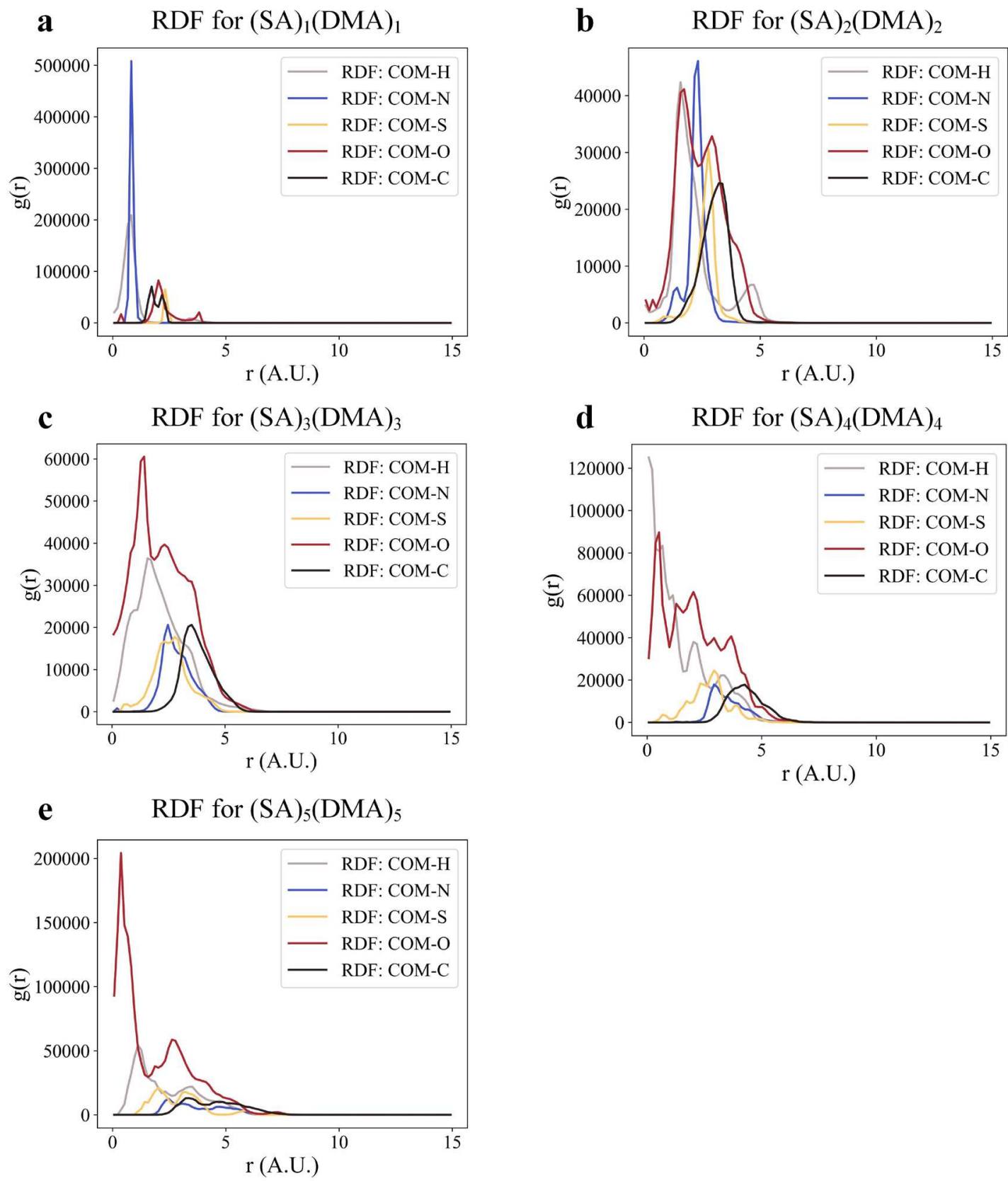
Extended Data Figure 8 | Cluster shape anisotropy distribution across all structures for cluster (SA) $)_{m}(D M A)_{n}(m=0 \sim 4, n \sim 0 \sim 4)$ at $278 \mathrm{~K}$.

DMA number

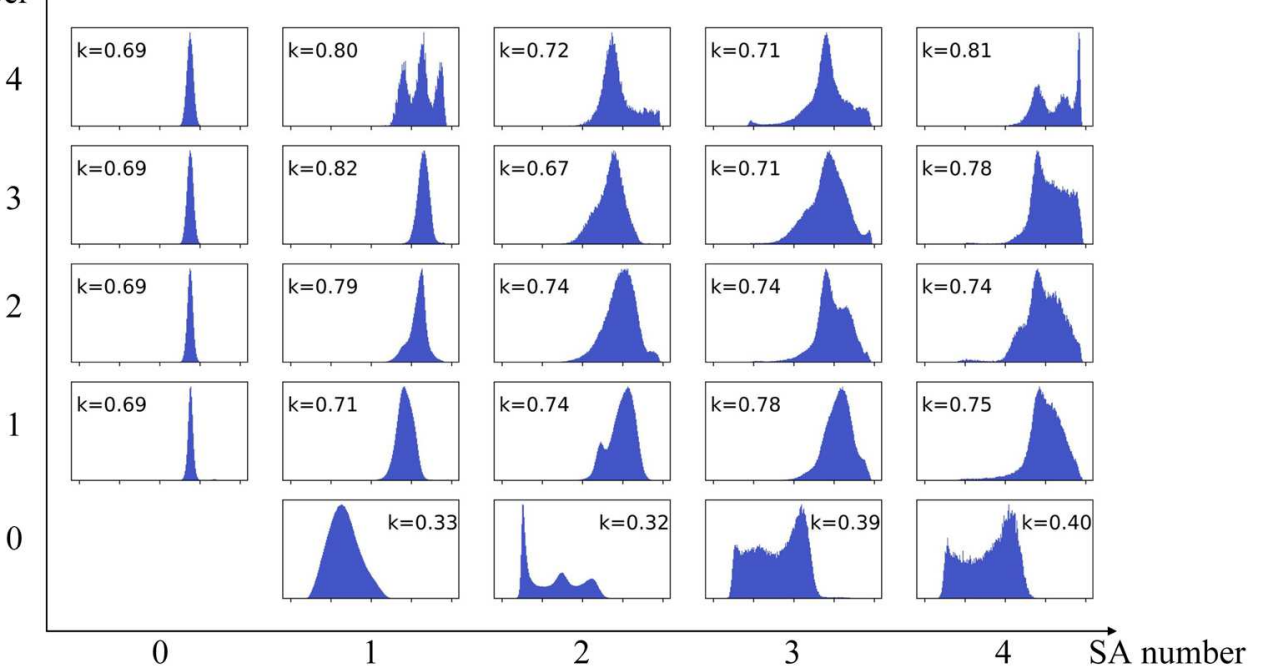

